\title{
Prospects for asteroseismology
}

\author{
Jørgen Christensen-Dalsgaard • Günter Houdek
}

(C) Springer-Verlag $\bullet \bullet \bullet \bullet$

\begin{abstract}
The observational basis for asteroseismology is being dramatically strengthened, through more than two years of data from the CoRoT satellite, the flood of data coming from the Kepler mission and, in the slightly longer term, from dedicated ground-based facilities. Our ability to utilize these data depends on further development of techniques for basic data analysis, as well as on an improved understanding of the relation between the observed frequencies and the underlying properties of the stars. Also, stellar modelling must be further developed, to match the increasing diagnostic potential of the data. Here we discuss some aspects of data interpretation and modelling, focussing on the important case of stars with solar-like oscillations.
\end{abstract}

Keywords asteroseismology; stellar internal physics; stellar structure; stellar evolution; helioseismology; solar composition

\section{Introduction}

In the last two decades data have become available on solar oscillations, which have allowed detailed inferences to be made on the properties of the solar interior (Christensen-Dalsgaard 2002). This has led to the determination of the solar sound speed with considerable accuracy, detailed tests of the equation of state of the solar interior and a determination of the solar internal rotation, the origin of which is still poorly understood.

Jørgen Christensen-Dalsgaard

Department of Physics and Astronomy, Aarhus University, DK-8000 Aarhus C, Denmark

Günter Houdek

Institute of Astronomy, University of Vienna, A-1180 Vienna, Austria
The inferences about the solar internal structure obviously provide a strong test of modelling of stellar evolution. Although earlier solar models were in reasonable, but far from perfect, agreement with the helioseismic determinations (Gough et al. 1996), recent redeterminations of the solar surface abundance have led to a very substantial increase in the discrepancies between the Sun and the model (for reviews, see Guzik 2006, 2008; Basu \& Antia 2008, see also Section 4.1).

The Sun is only one star at a specific stage of its evolution, and its structure is relatively simple, compared with other stars. Thus it is of obvious importance to extend seismic investigations to other stars. This is becoming possible, thanks to the development of groundbased instrumentation and the launch of three space missions that are providing extensive data on stellar oscillations. Thus it is important to consider the tools available for the analysis of such asteroseismic data and summarize the results that have already been obtained.

Here we concentrate on solar-like oscillations; these are characterized by being intrinsically damped and excited stochastically by near-surface convection (e.g., Houdek et al. 1999; Houdek 2006). Thus they are found in relatively cool stars, with significant outer convection zones, both on the main sequence and on the red-giant branch, as recently confirmed spectacularly by the CoRoT mission (De Ridder et al. 2009). The modes relevant to observations of distant stars have low spherical-harmonic degree and hence are generally high-order acoustic modes; however, in evolved stars they may take on a mixed character, with a component corresponding to an internal gravity mode in the deep interior of the star, very substantially enhancing their diagnostic value (e.g., Christensen-Dalsgaard 2004, see also Section 2.4).

At the simplest level, the oscillation frequencies are determined by the global properties of the star, more specifically the mean density $\bar{\rho} \propto M / R^{3}$, where $M$ is 
the mass and $R$ the surface radius of the star. When combined with additional, nonseismic, data and stellar modelling, the stellar radius can be determined with substantial precision and, one may hope, accuracy (Stello et al. 2009). Such a determination of the radius is particularly important for observations of extra-solar planets using the transit technique, where determination of the radius of the planet requires knowledge of the stellar radius (Kjeldsen et al. 2009). From the point of view of stellar modelling, however, it is of substantially greater interest to obtain information about the stellar interior. From low-degree acoustic modes a measure can be obtained that is sensitive to the properties of the stellar core and hence, with additional assumptions, provides a measure of the amount of hydrogen consumed during stellar evolution and thus of the stellar age. With sufficiently detailed and accurate observations additional information can be obtained about the stellar interior, including inferences of the soundspeed and density variation in the central parts of the star (e.g., Gough \& Kosovichev 1993; Basu et al. 2002; Roxburgh 2004).

An obvious requirement for asteroseismic inferences is the availability of good observational data. This is increasingly being met (see Section 3). Ground-based observations of Doppler velocity have been possible for a few stars, at level of sensitivity of a few $\mathrm{cm} \mathrm{s}^{-1}$. Space-based asteroseismic observations were started serendipitously by the WIRE satellite (Buzasi et al. 2005; Brunt 2007), followed by the launch in 2003 of the highly successful Canadian micro-satellite MOST (Walker et al. 2003; Matthews 2007). The French-led CoRoT satellite mission (Baglin et al. 2009), launched at the end of 2006, has provided photometric oscillation data on a large number of stars, and high-quality data on an even more extensive stellar sample is promised by the NASA Kepler mission launched in March 2009 (Borucki et al. 2009). Further observational projects promise a substantial increase in the quality of data available for asteroseismic analysis. However, the interpretation also depends on an adequate understanding of the relation between the observed oscillation properties, in particular the frequencies, and the underlying properties of the star, in order to design optimal diagnostics based on the observations. Furthermore, the physical interpretation of the results relies on stellar modelling, relating the physics of stellar interiors to stellar structure. Thus a crucial aspect of the analysis is the reliable computation of stellar models. This should obviously ensure numerical accuracy; an important step towards this goal is the detailed comparison of stellar models and oscillation frequencies computed by independent codes that has been undertaken in the ESTA collaboration, initially as part of the CoRoT project (Lebreton et al. 2008a, b; Mova et al. 2008). However, an equally important aspect is to ensure that the unavoidable approximations in the modelling are implemented in a consistent and accurate manner; only in this case can the asteroseismic analysis provide a reliable assessment of these approximations and, ideally, point to ways to improve them. Finally, the results should be presented in a statistically well-defined manner, such that the significance of the inferred properties, and of any deviations from the assumed models, can be assessed. It is probably fair to say that we are still some way from meeting these requirements.

In the present paper we discuss some aspects of the diagnostics of solar-like oscillations, based on asymptotic relations and computed properties; this in particular emphasizes the important probing of stellar cores. In addition, we consider some examples of uncertainties in stellar internal physics which have been, or may be, amenable to seismic inferences. More extensive overviews of helio- and asteroseismic diagnostics were provided by, for example, Gough (1993) and Christensen-Dalsgaard (2004), and a comprehensive discussion of asteroseismology can be found in Aerts et al. (2009).

\section{Asteroseismic diagnostics}

\subsection{Simple asymptotics of acoustic modes}

The observed oscillation modes in main-sequence solarlike stars are driven predominantly by the vigorous turbulence in the superficial stellar layers. This results in a rich acoustic pulsation spectrum with a characteristic comb-like frequency structure. In distant stars only low-degree modes can be observed; however, the observed modes are generally of high order allowing us to use asymptotic theory. For modes satisfying $l / n \rightarrow 0$, where $n$ is radial order and $l$ degree, the frequencies $\nu_{n l}$ can be estimated as Vandakurov 1967; Tassoul 1980; Gough 1986):

$$
\nu_{n l} \simeq\left(n+\frac{l}{2}+\epsilon\right) \nu_{0}-\frac{A L^{2}-B}{\nu_{n l}} \nu_{0}^{2}+\mathrm{O}\left(\nu_{0}^{4}\right),
$$

where

$\nu_{0}=\left[2 \int_{0}^{R} \frac{\mathrm{d} r}{c}\right]^{-1}$

is the inverse of twice the sound travel time between the centre and acoustic surface, $c$ is the sound speed, 
and

$A=\frac{1}{4 \pi^{2} \nu_{0}}\left[\frac{c(R)}{R}-\int_{0}^{R} \frac{\mathrm{d} c}{\mathrm{~d} r} \frac{\mathrm{d} r}{r}\right]$.

The frequency-dependent coefficient $\epsilon$ is determined by the reflection properties of the surface layers, as is the small correction term $B$ (e.g., Gough 1986), and $L=\sqrt{l(l+1)}$. The value of $\nu_{0}$ can be estimated from taking the average (over $n$ and $l$ ) of the so-called large frequency separation

$\Delta \nu_{n l} \equiv \nu_{n+1 l}-\nu_{n l}$

between modes of like degree and consecutive order. The resulting comb-like frequency structure consists of modes of odd degree falling approximately halfway between modes of even degree. The last two terms on the right-hand side of equation (1) lift the degeneracy between modes with the same value of $n+l / 2$, leading to the so-called small frequency separation $\delta \nu_{n l} \equiv$ $\nu_{n l}-\nu_{n-1} l+2$. The small frequency separation measures the extent to which the integrated history of nuclear transmutations has modified the structure of the energy-generating core. The mean small frequency separation, averaged over $n$ (indicated by angular brackets) becomes in the limit $l / n \rightarrow 0$

$\left\langle\delta \nu_{n l}\right\rangle=\left\langle\nu_{n l}-\nu_{n-1 l+2}\right\rangle \simeq 2 A(2 l+3) \frac{\nu_{0}^{2}}{\left\langle\nu_{n l}\right\rangle}$,

and is therefore proportional to the coefficient $A$ (Eq. 3). The coefficient $A$ measures predominantly the stratification of the energy-generating core through the gradient of the sound speed and hence is sensitive to the chemical composition there and consequently is an indicator for stellar age (e.g., Christensen-Dalsgaard 1984, 1993; Gough 1986, 2001; Ulrich 1986; Houdek \& Gough 2008).

From Eq. (11) it follows that, to this asymptotic order,

$D_{n l}=\frac{\nu_{n l}-\nu_{n-1 l+2}}{2 l+3}$

is a function of frequency. The same is true for the following ratio:

$D_{n l}^{(1)}=\frac{\left(\nu_{n-1 l}+\nu_{n l}\right) / 2-\nu_{n-1 l+1}}{l+1}$,

which is also a useful diagnostic, particularly when only modes of degree 0 and 1 can be identified from observations.

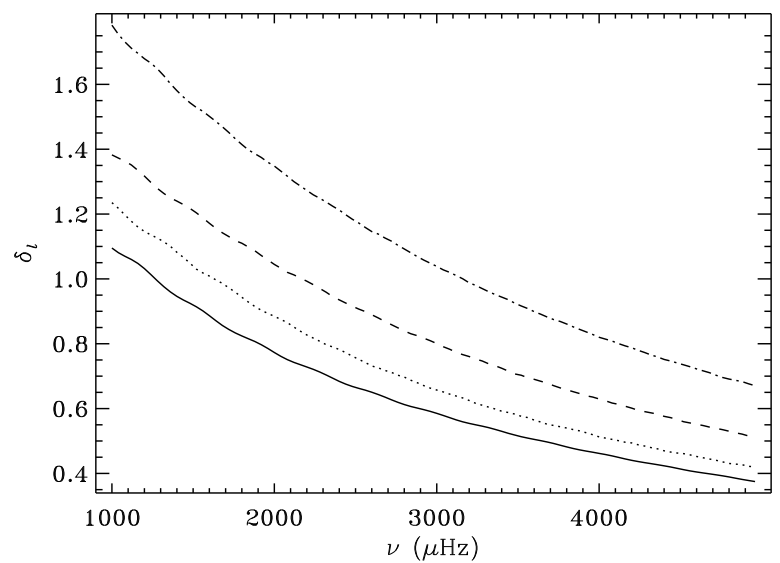

Fig. 1 The internal phase shift $\delta_{l}$ (cf. Eq. 8) for a model of the present Sun, as a function of cyclic frequency. Continuous curve: $l=0$; dotted curve: $l=1$; dashed curve: $l=2$; and dot-dashed curve: $l=3$.

\subsection{The inner phase shift}

Further insight into the diagnostic potential of lowdegree solar-like oscillations has been developed by Roxburgh and Vorontsov. They represent the effect of the structure of the core by an internal phaseshift $\delta_{l}(\omega)$ (Roxburgh \& Vorontsov 1994), defined such that the asymptotic behaviour of the acoustic modes can be expressed as

$\frac{(\rho c)^{1 / 2}}{r} p^{\prime} \simeq A_{\mathrm{p}} \sin \left(\omega \tilde{\tau}-\frac{\pi}{2} l+\delta_{l}\right)$,

where $\rho$ is density, $p^{\prime}$ is the Eulerian perturbation to pressure, $\omega=2 \pi \nu$ is angular frequency and

$\tilde{\tau}=\int_{0}^{r} \frac{\mathrm{d} r}{c}$

is the acoustic distance from the centre, corresponding to the radius $r$. They noted that $\delta_{l}$ can be computed as a function of frequency by matching partial solutions to the oscillation equations to the asymptotic expression in Eq. (8). We have implemented this by considering solutions satisfying both the central and surface boundary conditions, but allowing a discontinuity in the solution at a suitably chosen point near the surface. Figure 1 shows the results for a model of the present Sun.

From Eq. (8) and a similar asymptotic representation of the solution in the outer parts of the model, the oscillation frequencies can be obtained as

$\nu_{n l}=\nu_{0}\left[n+\frac{l}{2}+\pi^{-1}\left(\tilde{\alpha}\left(\omega_{n l}\right)-\delta_{l}\left(\omega_{n l}\right)\right)\right]$,

where $\tilde{\alpha}(\omega)$ is a surface phase function (see also Christensen-Dalsgaard \& Pérez Hernández 1992) which, 


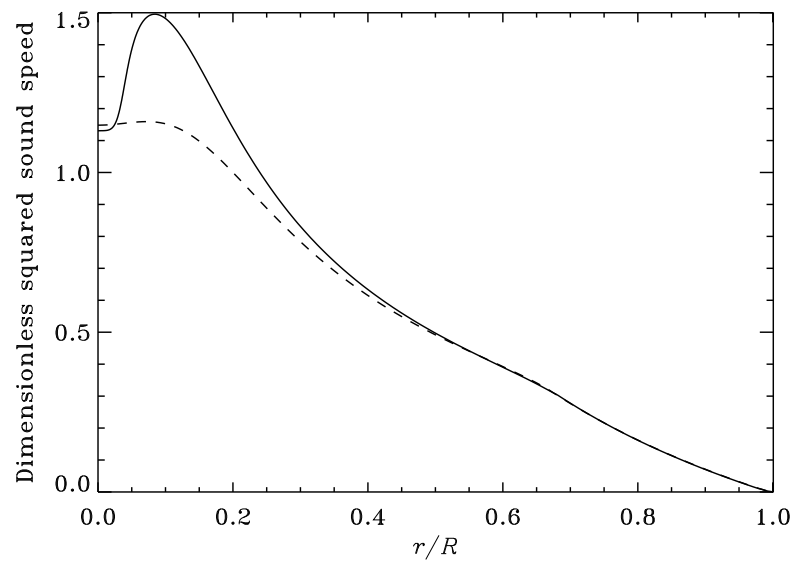

Fig. 2 Dimensionless squared sound speed, in units of $G M / R$, as a function of fractional radius, for models of $\alpha$ Cen A (solid) and B (dashed)

for low-degree modes, is independent of degree. This expression is clearly closely related to the simple asymptotic expression in Eq. (1) above, with the small frequency separation being related to $\delta_{l}$ through

$\nu_{n l}-\nu_{n-1 l+2}=\frac{\nu_{0}}{\pi}\left(\delta_{l+2}-\delta_{l}\right)$.

Roxburgh \& Vorontsov (1994) noted that, in the simplest asymptotic approximation, $\delta_{l}$ can be expressed as

$\delta_{l}(\omega)=\delta_{0}(\omega)+l(l+1) \hat{\delta}(\omega)$

where

$\hat{\delta} \simeq \frac{1}{2 \omega}\left[\frac{c(R)}{R}-\int_{0}^{R} \frac{\mathrm{d} c}{\mathrm{~d} r} \frac{\mathrm{d} r}{r}\right]$.

Given Eq. (11), this evidently leads to Eq. (5) for the average small separation, with $A$ given by Eq. (3).

The simple asymptotic behaviour in Eqs (11) and (3), or Eq. (12) and Eq. (13), assumes that the equilibrium structure is varying slowly with position. As a star evolves on the main sequence, the increase in the central mean molecular weight leads to a local decrease in the sound speed, as illustrated in Fig. 2 for the components of $\alpha$ Cen binary system. At the age of $6.98 \mathrm{Gyr}$ of the system the $\mathrm{B}$ component, with a mass of $0.928 \mathrm{M}_{\odot}$, is relatively unevolved, while the A component, with a mass of $1.111 \mathrm{M}_{\odot}$, is nearing the end of central hydrogen burning. The effect is evident in the difference in the behaviour of the dimensionless sound speed and is reflected in the scaled small separations shown in Fig. 3. While the scaled separations, in accordance with Eq. (1), are essentially just a function of frequency for
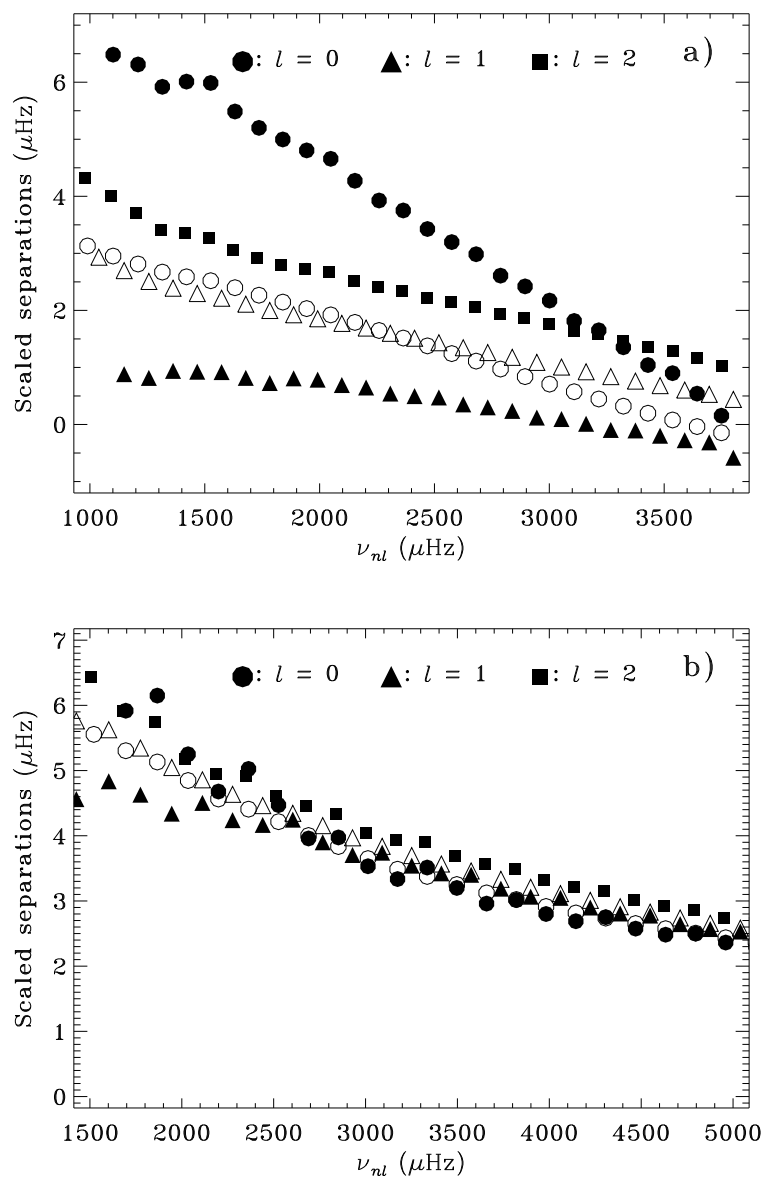

Fig. 3 Scaled small frequency separations (cf. Eqs 6 and 7), for models of $\alpha$ Cen A (panel a) and B (panel b). Filled symbols: $D_{n l}^{(1)}$, for $l=0,1$ and 2 ; the degree is indicated by the symbol, as shown in the figure. Open symbols: $D_{n l}$, for $l=0$ and 1 . Note that according to Eq. (1) (see also Eq. 5) these quantities are expected to be functions of frequency alone

$\alpha$ Cen B, for the A component there is very substantial scatter, reflecting the rapid variation of the sound speed in the core.

This behaviour is illustrated in Fig. 4, in terms of the scaled differences $[l(l+1)]^{-1}\left(\delta_{l}-\delta_{0}\right)$ in the internal phase shift, which according to Eq. (12) is expected to be a function of frequency. This is clearly approximately satisfied for the $\mathrm{B}$ component, whereas for the A component the results for $l=1$ deviate strongly from the expected asymptotic behaviour. Interestingly, the differences for $l=1$ show a distinct oscillatory behaviour which is related to the base of the convection zone (see also Roxburgh 2009, and Section 2.3).

It was noted by Roxburgh \& Vorontsov (2003) that the uncertainty in the modelling of the near-surface layers (see Section 2.5) has a significant effect on the small 

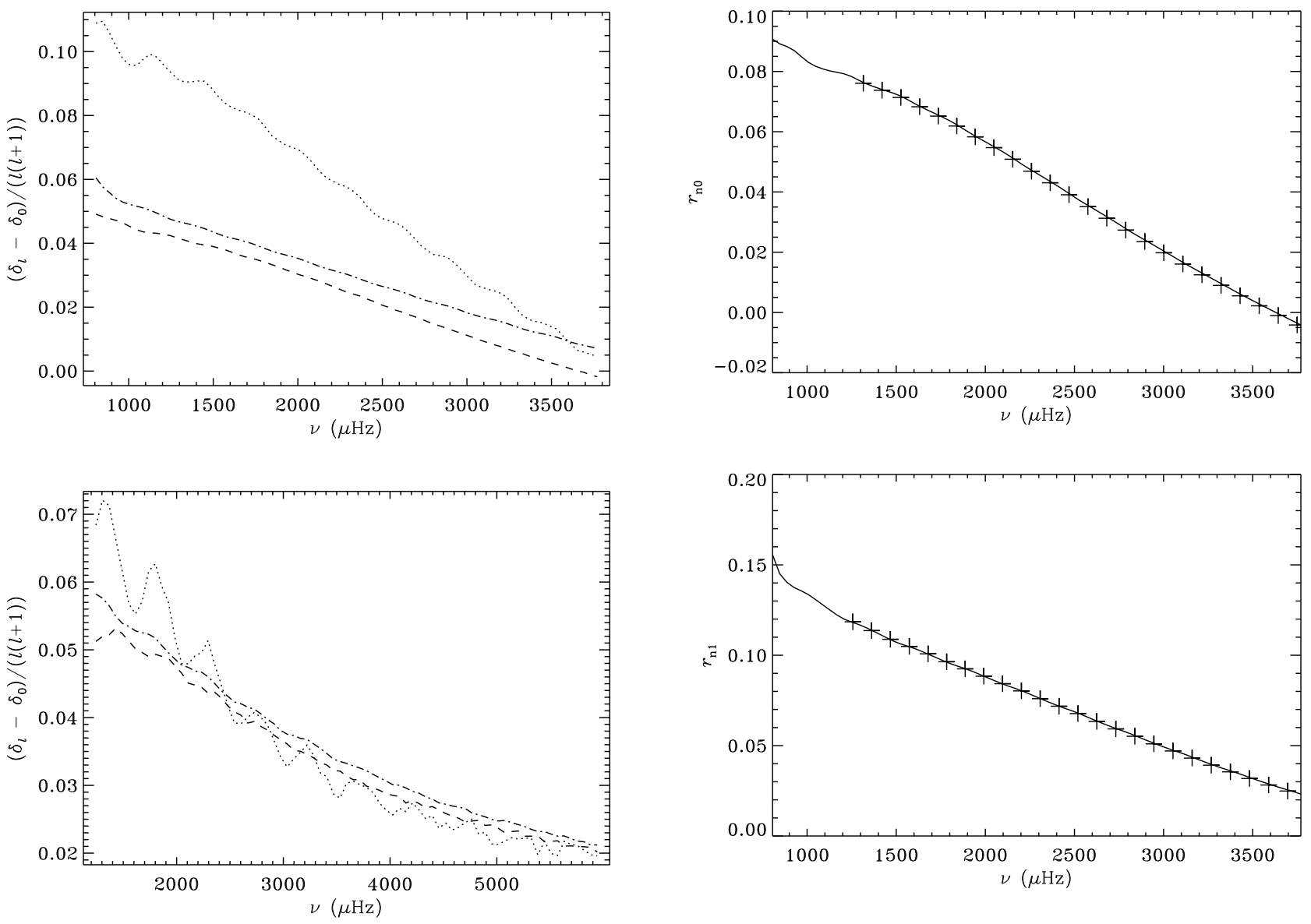

Fig. 4 Scaled differences in internal phase shift, for models of $\alpha$ Cen A (top) and B (bottom). Dotted curve: $l=1$; dashed curve: $l=2$; dot-dashed curve: $l=3$. Note that according to Eq. (12) these differences are expected to be functions of frequency alone

frequency separation. They pointed out that this effect is strongly suppressed by considering instead ratios such as

$$
\begin{aligned}
& r_{n 0}=\frac{\nu_{n 0}-\nu_{n-12}}{\nu_{n-11}-\nu_{n 1}} \\
& r_{n 1}=\frac{\nu_{n 1}-\nu_{n-13}}{\nu_{n 0}-\nu_{n+10}} .
\end{aligned}
$$

They also demonstrated that to this accuracy the ratios are related to the internal phase shifts by

$r_{n 0}=\left(\delta_{2}-\delta_{0}\right) / \pi, \quad r_{n 1}=\left(\delta_{3}-\delta_{1}\right) / \pi$.

This is illustrated in Fig. 5 for a model of $\alpha$ Cen A. The diagnostic potential of these separation ratios, and their insensitivity to even very substantial differences in the surface layers, was further analyzed by Otí Floranes et al. (2005) and Roxburgh (2005).

Fig. 5 Frequency-separation ratios (cf. Eq. 14 symbols) and difference $\left(\delta_{l+2}-\delta_{l}\right) / \pi$ in internal phase shifts (curve), for a model of $\alpha$ Cen A. Top: $l=0$, bottom: $l=1$.

One may hope that further analysis of the internal phase function can provide insight on the diagnostic potential of the frequency separations, beyond the simplest asymptotics. Roxburgh \& Vorontsov (1994) developed relatively simple expressions for $\delta_{l}$, in terms of the structure of the core, although these have apparently not been exploited in detail so far (see also Roxburgh \& Vorontsov 2000, 2007).

As discussed in Section 4.2 the diagnostics of convective cores is particularly important. The mixing in the core leads to sharp variations in composition and hence sound speed there, causing strong departures from the simple asymptotics of low-degree modes, as reflected in the inner phase shift and the small frequency separations. This is illustrated in Fig. 6 for a model resembling Procyon. A comparison with the results for the much more benign solar model, illustrated in Fig. 1. clearly shows the change in the behaviour of the inner phase shift $\delta_{l}$. This indicates the potential for using frequencies of low-degree modes to probe the properties of convective cores in stars showing solar-like 


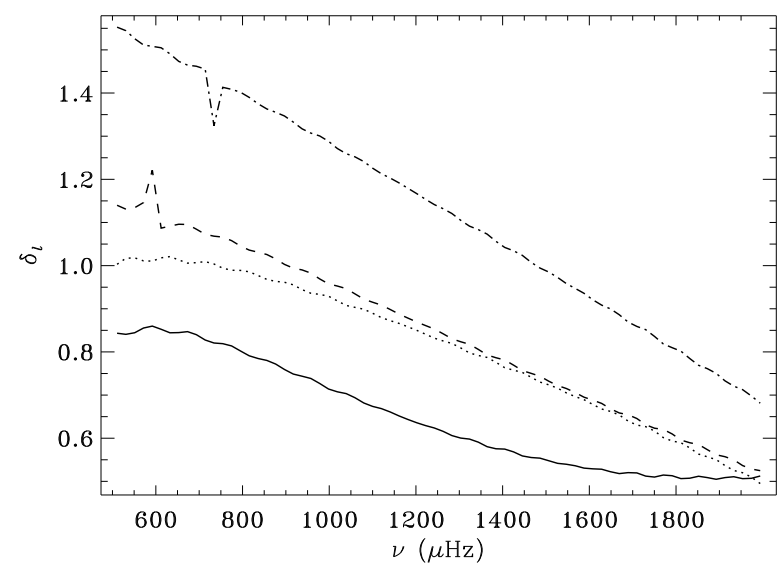

Fig. 6 The internal phase shift $\delta_{l}$ for a model approximating Procyon, with mass $M=1.5 \mathrm{M}_{\odot}$ and central hydrogen abundance $X_{\mathrm{c}}=0.05$. Continuous curve: $l=0$; dotted curve: $l=1$; dashed curve: $l=2$; and dot-dashed curve: $l=3$. The glitches for $l=2$ and 3 are probably associated with mixed modes (see Section 2.4)

oscillations (see also Popielski \& Dziembowski 2005). Cunha \& Metcalfe (2007) considered the specific case of a small growing convective core, deriving diagnostics, based on an asymptotic analysis of the oscillations, of the properties of the core. They found that the quantity

$\Delta_{\mathrm{CM}}=\frac{D_{n 0}}{\Delta \nu_{n-11}}-\frac{D_{n 1}}{\Delta \nu_{n 0}}$

(see Eqs 4 and 6) provides a measure of stellar age; more specifically, it is sensitive to the discontinuity in the sound speed resulting from the composition discontinuity at the edge of the core.

\subsection{Diagnostics of acoustic glitches}

The asymptotic expression (11) is valid only if the spatial scale of variation of the equilibrium state is everywhere much greater than the inverse vertical wavenumber of the mode. But that condition is not satisfied in the Sun and main-sequence solar-like stars: there is small-scale variation associated with ionization of abundant elements and the near discontinuity in low derivatives of the density at the base of the convection zone. These abrupt variations induce small-amplitude oscillatory components (with respect to frequency) in the spacing of the cyclic eigenfrequencies $\nu_{n l}$ of seismic oscillation and consequently also in $\Delta \nu_{n l}$ and $\delta \nu_{n l}$. We call such abrupt variations an acoustic glitch.

A convenient and easily evaluated measure of the oscillatory component produced by acoustic glitches is the second multiplet-frequency difference with respect
Table 1 Seismically determined properties of acoustic glitches in the Sun (using BiSON data; Basu et al. 2007) and in Model S (Christensen-Dalsgaard et al. 1996) based on the analysis by Houdek \& Gough (2007) of low-degree modes $(l=0, \ldots, 3): \delta \gamma_{1} /\left.\gamma_{1}\right|_{\text {II }}$ and $\tau_{\text {II }}$ are the relative depression of the first adiabatic exponent and the acoustic depth of the second helium ionization zone, and $\tau_{\mathrm{c}}$ is the acoustic depth of the sharp transition from radiative to convective heat transport at the base of the convection zone

\begin{tabular}{lccc}
\hline & $-\delta \gamma_{1} /\left.\gamma_{1}\right|_{\text {II }}$ & $\tau_{\text {II }}(\mathrm{s})$ & $\tau_{\mathrm{c}}(\mathrm{s})$ \\
\hline Sun & 0.043 & 819 & 2310 \\
Model S & 0.045 & 815 & 2270 \\
\hline
\end{tabular}

to order $n$ amongst modes of like degree $l$ :

$\Delta_{2} \nu_{n l} \equiv \nu_{n-1 l}-2 \nu_{n l}+\nu_{n+1 l}$

(Gough 1990). Any localized region of rapid variation of either the sound speed $c$ or the density scale height, or a spatial derivative of them, induces an oscillatory component in $\Delta_{2} \nu$ (the subscripts $n l$ have been dropped) with a 'cyclic frequency' approximately equal to twice the acoustic depth

$\tau=\int_{r_{\text {glitch }}}^{R} c^{-1} \mathrm{~d} r$

(not to be confused with the acoustic radius, Eq.9) of the glitch, and with an amplitude which depends on the amplitude of the glitch and which decays with $\nu$ once the inverse radial wavenumber of the mode becomes comparable with or less than the radial extent of the glitch.

Various approximate formulae for the oscillatory components that are associated with the helium ionization have been suggested and used, by e.g., Basu et al. (1994); Basu \& Antia (2004), Monteiro \& Thompson (1998, 2005), and Gough (2002), not all of which are derived directly from explicit acoustic glitches. Gough used an analytic function for modelling the dip in the first adiabatic exponent $\gamma_{1}=(\partial \ln p / \partial \ln \rho)_{s}$, where $p, \rho$ and $s$ are pressure, density and specific entropy. In contrast, Monteiro \& Thompson assumed a triangular form. Basu et al. have adopted a seismic signature for helium ionization that is similar to that arising from a single discontinuity; the artificial discontinuities in the sound speed and its derivatives that this and the triangular representations possess cause the amplitude of the oscillatory signal to decay with frequency too gradually, although that deficiency may not be immediately noticeable within the limited frequency range in which adequate asteroseismic data are or will imminently be available. More recently Houdek \& Gough (2007) proposed a seismic diagnostic in which the variation of the first adiabatic exponent $\gamma_{1}$ in the helium 
ionization zone is represented with a pair of Gaussian functions. This correctly results in a decay of the amplitude of the seismic signature with oscillation frequency that is faster than that which the triangular and the single-discontinuity approximations imply, and also takes some account of the two ionization states of helium. Moreover, Houdek \& Gough (2007) incorporated the acoustic cutoff frequency into the variation of the eigenfunction phase with acoustic depth $\tau$, thereby improving the discrepancy between the seismically inferred depths of the acoustic glitches and that of a corresponding stellar model.

Fig. 7 shows the outcome of fitting Houdek \& Gough's (2007) seismic diagnostic to BiSON data (top panel), together with the individual contributions from the acoustic glitches (lower panel). The individual contributions provide a measure of the properties of the acoustic glitches in the background stratification of the star. One might hope that the variation of the sound speed $c$ induced by helium ionization might enable one to determine from the low-degree eigenfrequencies a measure that is directly related to, perhaps even almost proportional to, the helium abundance, with little contamination from other properties of the structure of the star. Table 1 compares some of the properties of the acoustic glitches obtained from the BiSON data, and from adiabatically computed eigenfrequencies of Model S (Christensen-Dalsgaard et al. 1996), using the analysis by Houdek \& Gough (2007). The values between the Sun and Model S differ by less than $3 \%$, demonstrating the diagnostic potential of this seismic analysis using only low-degree modes.

\subsection{Mixed modes}

We have so far considered only modes that are predominantly of acoustic nature; these $p$ modes are expected at the relatively high frequencies where the stochastic excitation is most efficient. However, in evolved stars, beyond the end of central hydrogen burning, internal gravity waves, or $g$ modes, reach frequencies in the range of the stochastically excited modes. Their properties are characterized by the buoyancy, or Brunt-Väisälä, frequency $N$, determined by

$$
N^{2}=g\left(\frac{1}{\gamma_{1}} \frac{\mathrm{d} \ln P}{\mathrm{~d} r}-\frac{\mathrm{d} \ln \rho}{\mathrm{d} r}\right) \simeq \frac{g^{2} \rho}{p}\left(\nabla_{\mathrm{ad}}-\nabla+\nabla_{\mu}\right),
$$

where $g$ is the gravitational acceleration. The last equality assumes the ideal gas law; $\nabla=\mathrm{d} \ln T / \mathrm{d} \ln p$, $\nabla_{\text {ad }}$ is its adiabatic value, and $\nabla_{\mu}=\mathrm{d} \ln \mu / \mathrm{d} \ln p$, where $\mu$ is the mean molecular weight. In evolved stars $g$ becomes very high near the compact core; an additional

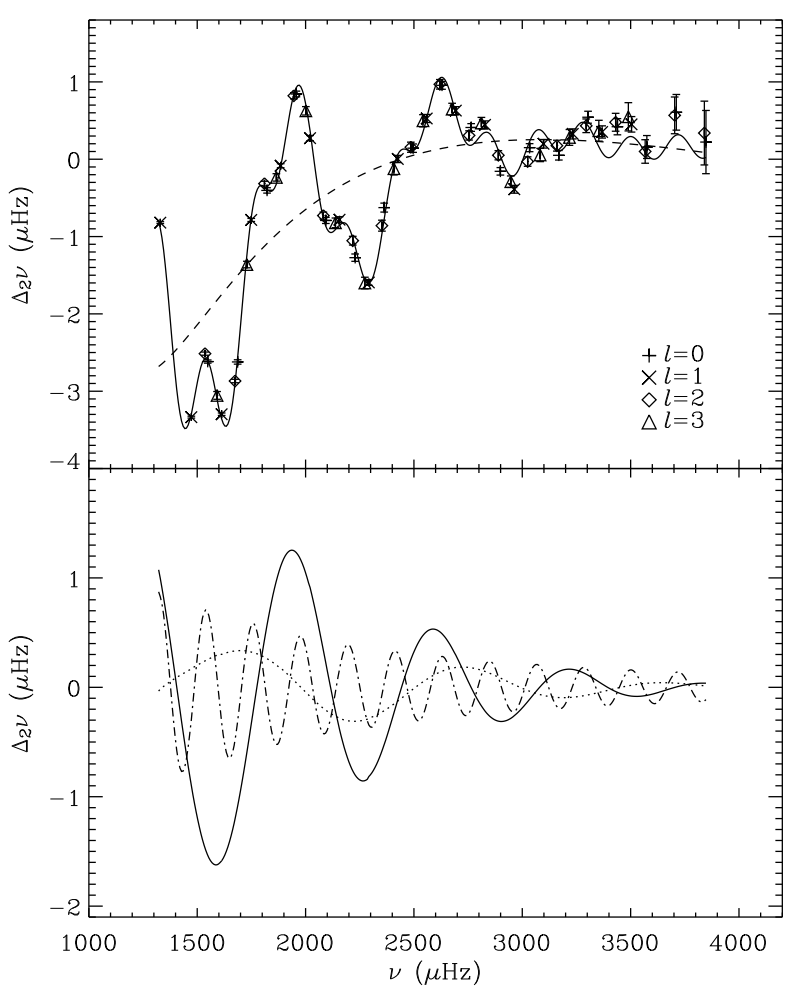

Fig. 7 Top: Symbols show second differences $\Delta_{2} \nu_{n l}$ (Eq.17) for low-degree modes of BiSON data collected over a period of 4752 days (Basu et al. 2007). The solid curve is Houdek \& Gough's (2007) seismic diagnostic, determined from fitting it to the data by least squares. The dashed curve is a smooth contribution of $\Delta_{2} \nu_{n l}$, modelled as a third-order polynomial in $\nu^{-1}$ guided by the asymptotic expression (11), and represents near-surface effects. Bottom: Individual contributions of the seismic diagnostic. The solid curve displays the contribution of the second ionization stage of the helium, and the dotted curve the first ionization stage of helium. The dot-dashed curve is the contribution from the discontinuity in the second density derivative at the base of the convection zone

contribution comes from $\nabla_{\mu}$ which is large in the region of varying hydrogen abundance left behind after nuclear fusion. As a result, $N$ may reach very high values in the central parts of the star.

As an example we consider a model of the subgiant $\eta$ Boo, where for the first time individual frequencies of solar-like oscillations in another star were identified (Kjeldsen et al. 1995). This is based on modelling of the star by Di Mauro et al. (2003); the model has a mass of $1.7 \mathrm{M}_{\odot}$ and a heavy-element abundance $Z=0.04$. Figure 8 shows the buoyancy frequency at a model age of $2.34 \mathrm{Gyr}$. It is evident that it reaches frequencies far higher than the acoustic cut-off frequency, around $1150 \mu \mathrm{Hz}$, in the atmosphere of the model which defines the upper limit to the frequencies of trapped acoustic modes. Also shown are the acoustic, or Lamb, 


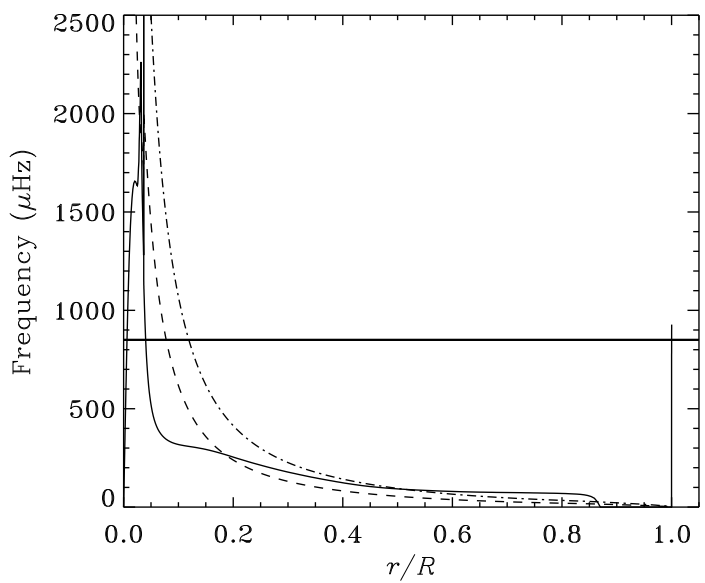

Fig. 8 Characteristic frequencies for a model of $\eta$ Boo. The solid curve shows $N / 2 \pi$ where $N$ is the buoyancy frequency (cf. Eq. 19), and the dashed and dot-dashed curves show $S_{l} / 2 \pi$ for $l=1$ and 2 , respectively. The heavy horizontal line marks a typical observed frequency of $\eta$ Boo (see Di Mauro et al. 2003)

frequencies $S_{1}$ and $S_{2}$, defined by

$S_{l}^{2}=\frac{l(l+1) c^{2}}{r^{2}}$.

The properties of a mode of oscillation are controlled by the magnitude of the frequency relative to $S_{l}$ and $N$. In the region of the star where the frequency exceeds $N$ and $S_{l}$ the mode behaves predominantly as an acoustic wave. Where the frequency is below $N$ and $S_{l}$ the mode has the character of an internal gravity wave. Finally, in the so-called evanescent region, where the frequency is between $N$ and $S_{l}$, the mode amplitude varies exponentially with position, either increasing or decreasing.

In Fig. 8 the horizontal line marks a typical observed frequency in $\eta$ Boo. It is evident that this mode may have g-mode behaviour in the core of the model and p-mode behaviour in the envelope. The dominant behaviour depends on the variation in the evanescent region. If the eigenfunction decreases exponentially with $r$ in this region the mode has predominantly the character of a g mode, whereas if it increases exponentially, the mode has the character of a $\mathrm{p}$ mode. The discrimination between the two cases depends on the strength of the exponential variation and hence on the extent of the evanescent region. Thus the separation between the two cases is stronger for $l=2$ than for $l=1$ modes.

With the evolution on the sub-giant branch the star expands, and hence the acoustic frequencies decrease approximately as $\bar{\rho}^{1 / 2}$. On the other hand, the increasing central condensation increases the buoyancy
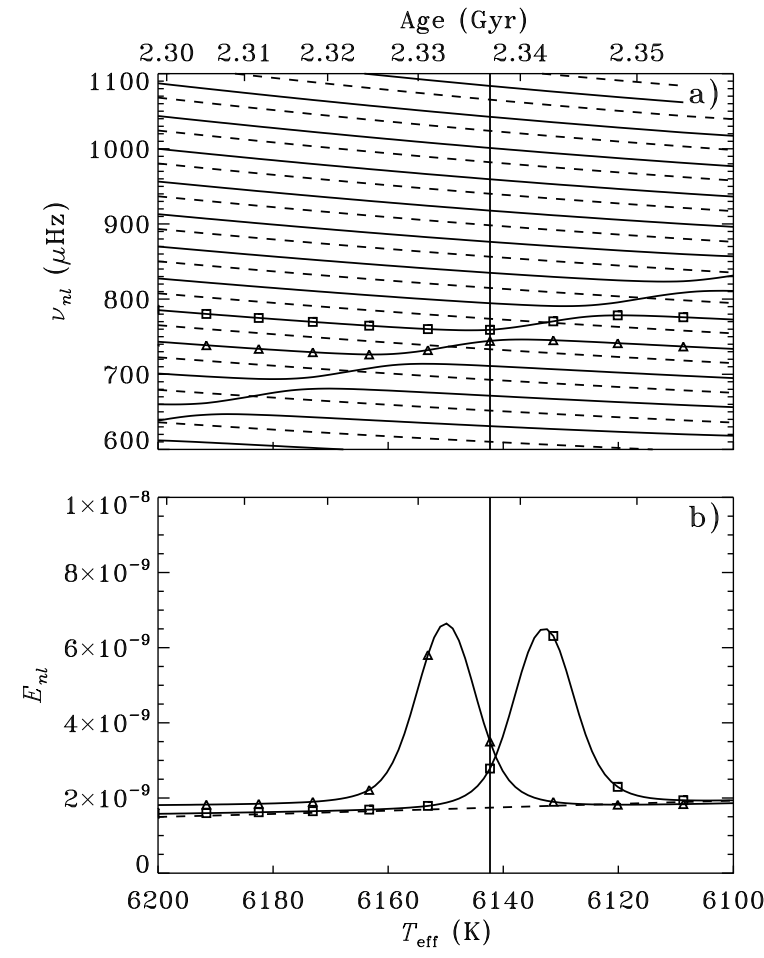

Fig. 9 Evolution of oscillation properties with age (upper abscissa) and effective temperature (lower abscissa) in a model of $\eta$ Boo. Panel (a) shows frequencies of modes with $l=0$ (dashed curves) and $l=1$ (solid curves). In panel (b) the solid curves show the evolution of the dimensionless inertia $E$ (cf. Eq. 21) for the two $l=1$ modes marked by triangles and squares in panel (a), and of a neighbouring mode with $l=0$ (dashed curve). The heavy vertical line identifies the model providing the best fit to the observed frequencies.

frequency and hence the frequencies of the $\mathrm{g}$ modes. The combined effect for modes of degree $l=0$ and 1 , in models near the identified stage of evolution of $\eta$ Boo, is illustrated in Fig. 9. For the radial modes, with $l=0$, and the predominantly acoustic $l=1$ modes the frequencies decrease with increasing age and hence decreasing effective temperature. However, there is evidently also a branch of frequencies, corresponding to a mode of predominantly g-mode character, increasing with age. Where this meets a predominantly acoustic mode, the two modes undergo an avoided crossing (Osaki 1975), where the frequencies approach quite closely without actually crossing. An example occurs at the vertical line, marking the model identified as best fitting the observations. At closest approach, the modes have a fully mixed nature, with similar amplitudes (in 

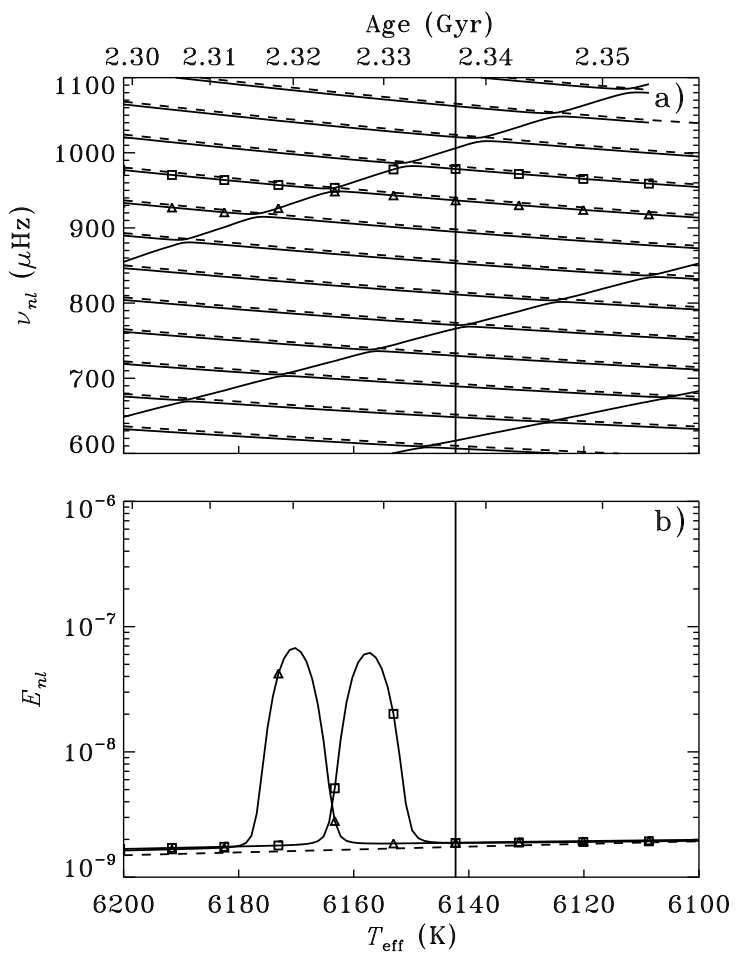

Fig. 10 As Fig. 9] but for $l=2$.

terms of energy density) in the central g-mode region and the p-mode envelope.

The variation in the physical nature of the modes can be followed by considering the normalized mode inertia

$E=\frac{\int_{V} \rho|\boldsymbol{\delta} \boldsymbol{r}|^{2} \mathrm{~d} V}{M\left|\boldsymbol{\delta} \boldsymbol{r}_{\mathrm{phot}}\right|^{2}}$,

where $\boldsymbol{\delta} \boldsymbol{r}$ is the displacement vector, $\boldsymbol{\delta} \boldsymbol{r}_{\text {phot }}$ is its photospheric value, and integration is over the volume $V$ of the star. This is illustrated in panel (b) of Fig. 9] for two modes with $l=1$ undergoing an avoided crossing, and for a neighbouring radial mode. For the latter, a purely acoustic mode, $E$ varies slowly with age. The $l=1$ modes have $E$ close to the radial modes when they behave as acoustic modes, while $E$ is somewhat higher where the modes have substantial g-mode character. As is clear from the figure the two modes exchange character at the avoided crossing; at the closest approach, near the vertical line, $E$ is the same in the two modes.

The corresponding results for $l=2$ are shown in Fig. 10. Here the number of g-mode branches is higher. Again, these undergo avoided crossings with the acoustic modes, but the minimum separation is so small that the avoided nature is barely discernible. Also, the mode inertias, shown in panel (b) of the figure, on the gmode branches exceed those of the radial modes by almost two orders of magnitude (note the logarithmic scale). The reason for this difference in behaviour between $l=1$ and 2 is the greater extent of the evanescent region in the latter case (cf. Fig. 8). This decreases the coupling between the g-mode and acoustic regions, making the g modes much more strongly trapped in the innermost parts of the model, and decreasing the interval over which the avoided crossing take place (see also Christensen-Dalsgaard 1980).

Oscillations with g-mode character are much more sensitive to the properties of stellar cores than are the purely acoustic modes. In particular, Eq. (19) shows that the buoyancy frequency depends directly on the gradient in the hydrogen abundance. Thus such mixed modes have a very substantial asteroseismic diagnostic potential. An important issue is whether the modes can be expected to be excited to observable amplitudes. It is perhaps not unreasonable to assume that the stochastic excitation excites the modes to a total energy that depends predominantly on the properties of the oscillations in the near-surface layers, where both the excitation and the dominant damping take place. In these superficial layers the characteristic timescale of the convection is of the same order of magnitude as the pulsation period of the oscillations (e.g. Goldreich \& Keeley 1977; Christensen-Dalsgaard \& Frandsen 1983). This leads to a strong coupling between the global oscillations and the turbulent velocity field and consequently efficient mode excitation by the convection (e.g. Balmforth 1992b; Houdek 2006). Since the properties of the oscillations in this region, for low-degree modes, are mainly determined by the frequency, we would therefore expect that the mode energy $\mathcal{E}=\mathcal{E}(\nu)$ is also a function of frequency. Using that $\mathcal{E} \sim E M\left\langle V^{2}\right\rangle$ (cf. Eq. 21), where $\left\langle V^{2}\right\rangle$ is the mean square amplitude, we might therefore expect

$\left\langle V^{2}\right\rangle^{1 / 2} \propto E^{-1 / 2}$,

at a given frequency. Based on Figs 9 and 10 we would therefore predict that for $l=1$ even modes on the $\mathrm{g}$ mode branch would have amplitudes that could be observable, whereas for $l=2$ modes with some g-mode character would only be visible in the narrow regions around the avoided crossings, for this model 1

\footnotetext{
${ }^{1} \mathrm{~A}$ more careful analysis of the visibility of the modes in terms of the peak height in the power spectra, rather than the root-meansquare velocity, shows a somewhat more complex picture, with strong dependence on the mode lifetime in relation to the duration of the observations; see Fletcher et al. (2006), Dupret et al. (2009).
} 


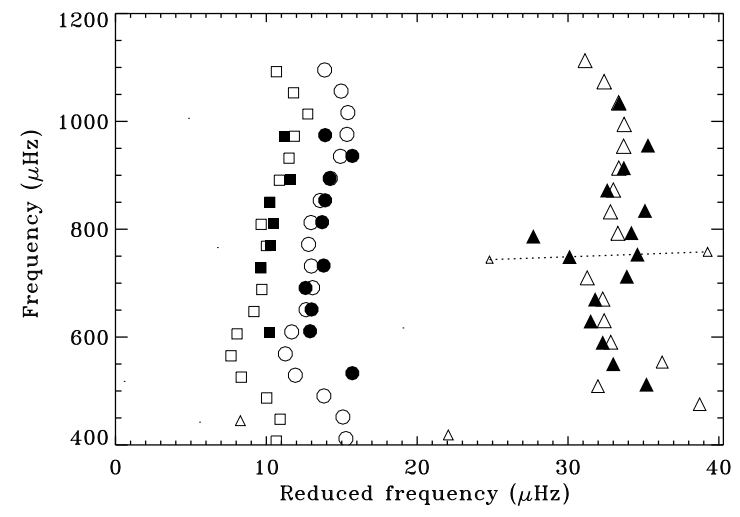

Fig. 11 Échelle diagram (see text), with a starting frequency of $33.7 \mu \mathrm{Hz}$ and a large separation $\Delta \nu=40.3 \mu \mathrm{Hz}$. Modes of degree $l=0,1$ and 2 are shown with circles, triangles and squares, respectively. Open symbols show results for the best-fitting model of $\eta$ Boo (Di Mauro et al. 2003), marked by a vertical line in Figs 9 and 10 while filled symbols are for combined observations of Kjeldsen et al. (2003) and Carrier et al. 2005).

It is of great interest to compare these model properties with the observed frequencies. We base this on a re-analysis by H. Kjeldsen (unpublished) of the observations by Kieldsen et al. (2003) and Carrier et al. (2005) (see Aerts et al. 2009, for details). The results are illustrated in an échelle diagram (see Grec et al. 1983) in Fig. 11] Here the frequency spectrum has been divided into slices of a length given by the large separation $\Delta \nu$, starting at a frequency of $33.7 \mu \mathrm{Hz}$. These reduced frequencies are plotted against the starting frequency of each slice, corresponding, in the figure, essentially to stacking the slices. According to the asymptotic expression for acoustic modes, Eq. (1), one would expect the result to be nearly vertical sequences of points, separated by the small separation, although perhaps with some variation arising from the acoustic glitch associated with the second helium ionization zone (cf. Section 2.3). For the observations, shown by filled symbols, this is indeed satisfied by the frequencies for $l=0$ and 2 . However, the points for $l=1$ clearly show a less regular behaviour.

To interpret these results the open symbols show corresponding results for a model matched to the observed frequencies, as well as the effective temperature and luminosity of the star; this model corresponds to the vertical lines in Figs 9 and 10 . The frequencies were corrected for near-surface effects according to the procedure of Kjeldsen et al. (2008) (see Section 2.5). To provide some indication of the visibility of the modes, the size of the symbols has been scaled to reflect the amplitude estimate in Eq. (22), relative to a radial mode of the same frequency. For $l=2$ this essentially makes the modes on the g-mode branches invisible, owing to their large $E$. However, the $l=1$ modes are retained, with a behaviour showing strong departures from the acoustic asymptotic relation, for modes with a strong mixed nature. As an example, the dotted line connects the two modes shown to undergo an avoided crossing in Fig. 9. as expected for modes at the closest point of an avoided crossing these are distributed equally on either side of the expected location of the acoustic mode. Although there is no detailed agreement between the observed and computed frequencies of the $l=1$ modes, the strong resemblance between their behaviour suggests that the observations show evidence for mixed modes in this star.

\subsection{Superficial problems}

A major issue in the analysis of solar-like oscillation frequencies is the influence of the near-surface layers. Stellar modelling normally treats these layers in a highly simplified manner, with a simple model of the stellar atmosphere and a simplified treatment of the upper, superadiabatic, parts of the convection zone, such as the mixing-length formulation (Böhm-Vitense 1958). Also, the effects of the turbulent Reynolds stresses on the structure are almost always ignored. In computations of oscillation frequencies the adiabatic approximation is commonly adopted, and perturbations to the turbulent Reynolds stresses are neglected. When nonadiabatic calculations are carried out the treatment of the perturbation to the convective flux is a major uncertainty in the calculation (e.g., Baker \& Gough 1979; Gough 1980; Balmforth 1992a).

These problems are confined to the stellar atmosphere and the outermost part of the the stellar interior. In these regions the properties of modes of low or intermediate degree are largely independent of degree, at least in spherically symmetric models, and hence the influence of the near-surface problems on the oscillation frequency is essentially just a function of frequency (e.g., Christensen-Dalsgaard \& Thompson 1997); for modes of intermediate degree there is some dependence on degree in the form of a trivial scaling with mode inertia. In the solar case, where modes over a broad range of degree are observed, these properties allow the near-surface errors in the model to be isolated and suppressed in the analysis of the observed frequencies. In fact, for models such as Model S they dominate the differences between the solar and model frequencies (e.g., Christensen-Dalsgaard et al. 1996). This provides an estimate of the effects in the solar case, showing that they are very small at low frequency and increase 
rapidly with frequency, as indeed expected from simple analyses (e.g., Christensen-Dalsgaard \& Gough 1980). As a consequence, they also have a substantial effect on the determination of the large frequency separation (see Eq. 4).

Such a separation is not possible for stellar observations, restricted to low degree; hence the near-surface effects must be kept in mind as a source of systematic error in the analysis. As discussed in Section 2.2 the effects are strongly suppressed in separation ratios (cf. Eq. 14), which are essentially determined just by the inner phase shift. This is extremely valuable for the diagnostics of stellar cores. To obtain further information from the observed frequencies, additional assumptions, so far with limited justification, must be made. The behaviour of the oscillations in the stellar atmosphere is largely controlled by the value of the frequency, measured in terms of the atmospheric acoustical cut-off frequency $\omega_{\mathrm{ac}}$ which, in the approximation of an isothermal atmosphere, is given by

$\omega_{\mathrm{ac}}=\frac{c}{2 H}$,

where $H$ is the pressure scale height (note that in an isothermal atmosphere with constant mean molecular weight the pressure scale height is equal to the density scale height). Thus, for stars similar to the Sun one can perhaps use the ansatz that the near-surface effects can be approximated by $a f_{\text {surf }}^{(\odot)}\left(\omega / \omega_{\text {ac }}\right)$ where $f_{\text {surf }}^{(\odot)}$ is obtained from the solar observations and $a$ is a constant to be determined as part of the fit of the model to the observed frequencies. In a similar spirit Kieldsen et al. (2008) suggested to represent the nearsurface frequency shift as $a\left(\omega / \omega_{0}\right)^{b}$, where $\omega_{0}$ is a suitable reference frequency, the exponent $b$ is obtained from the solar data, and $a$ is obtained from the frequency fit, together with a scaling factor that accounts for the difference in mean density between the star and the model.

It is obvious that these simple procedures have relatively weak justification, particularly for stars that are somewhat different from the Sun. Analysis of the detailed data for a broad range of stars that are being obtained by the Kepler mission will certainly contribute to a better characterization of the near-surface effects. Also, an overview of how these effects influence the frequencies of stellar models, depending on the stellar parameters, would be very interesting.

A key goal is clearly to improve the treatment of the physics of the near-surface regions. In the solar case, models of the near-surface region based on hydrodynamical simulations result in frequencies in better, but still not complete, agreement with the observations (Rosenthal et al. 1999; Li et al. 2002). Such calculations were also applied with apparent success to the analysis of MOST observations of $\eta$ Boo by Straka et al. (2006). The effects of convection dynamics on the oscillation frequencies were also discussed by Balmforth (1992b), Rosenthal et al. (1995) and Houdek (1996), who used the nonlocal, time-dependent formulation by Gough (1977a, b) in their linear stability analyses. These authors reported that the inclusion of the Reynolds stress in an equilibrium solar model reduces substantially the frequency difference between observed and adiabatically computed radial eigenfrequencies. This difference, however, becomes larger again when nonadiabaticity and convection dynamic effects are included in the stability computations, thereby compensating nearly the frequency shifts arising from the Reynolds stress in the equilibrium model. A similar conclusion was reported by Christensen-Dalsgaard et al. (1995) for $\eta$ Boo. The effect of such modelling on other stars should obviously be investigated.

\section{The observational situation}

The tiny amplitudes of solar-like oscillations have greatly complicated their observation in distant stars, despite extensive efforts. It was only the observations of Kjeldsen et al. (1995) that led to the first detection of individual modes of oscillations in a solarlike star, the star $\eta$ Boo; this was later confirmed by Kieldsen et al. (2003) and Carrier et al. (2005). However, in the last decade the development in observational techniques to measure Doppler velocity, largely motivated by the search for extra-solar planetary systems, has led to fairly detailed observations of solar-like oscillations in a number of stars (for a brief review, see Bedding \& Kjeldsen 2008), reaching a noise level in the amplitude spectrum as low as $1.4 \mathrm{~cm} \mathrm{~s}^{-1}$ Kieldsen et al. 2005). From space, photometric observations allow simultaneous observations of large numbers of stars. The CoRoT mission Baglin et al. 2009) has produced remarkable asteroseismic results (e.g., Michel et al. 2008a,b), including striking results on solar-like pulsations in red giants (e.g., De Ridder et al. 2009). Also, the first results from the Kepler mission (Borucki et al. 2009) confirm the great potential of the mission for asteroseismology (e.g., Christensen-Dalsgaard et al. 2007).

The observations generally provide a reliable measure of the large frequency separation (see Eq. 4). To move beyond this an identification of at least the degree of the modes is required. In the case, for example, of the binary system $\alpha$ Cen A and B Bouchy \& Carrier 
2001, 2002; Butler et al. 2004; Bedding et al. 2004; Bazot et al. 2007; Carrier \& Bourban 2003; Kieldsen et al. 2005) or for $\beta$ Hydri (Bedding et al. 2007) an unambiguous identification has been possible, based on a match to the asymptotic behaviour (cf. Eq. 1). This has led to detailed asteroseismic analyses for these stars, yielding precise determinations of their overall parameters and some indications of possible problems in the modelling (e.g., Eggenberger et al. 2004; Miglio \& Montalbán 2005).

In other cases, however, the mode identification has proved to be difficult. An interesting example is the star Procyon A ( $\alpha \mathrm{CMi}$ ), of spectral type F5IV-V, which has been the target of several asteroseismic observing campaigns. Evidence for a power excess associated with solar-like oscillations was found already by Brown et al. (1991); however, several subsequent observations have failed to make an unambiguous identification of the modes of oscillation, and doubt was even raised on the reality of the oscillations on the basis of observations from the MOST satellite (Matthews et al. 2004) (see, however, Bedding et al. 2005). A very extensive observing campaign, involving 11 telescopes, was carried out by Arentoft et al. (2008). This confirmed beyond any doubt that Procyon exhibited solar-like oscillations; the mode identification has proven to be difficult but is now close to being completed (Bedding et al., in preparation). Similar problems have been found in the analysis of data from the CoRoT satellite, such as the star HD49933, of spectral type F2V (Appourchaux et al. 2008; Benomar et al. 2009; Gaulme et al. 2009).

These problems in the data analysis appear to a large extent to be associated with the mode lifetime in these stars, which is substantially shorter than in, for example, the Sun or $\alpha$ Cen A and B, hence leading to a more complex spectrum of oscillations. Thus it is of substantial interest that a close relation has been found between the effective temperature $T_{\text {eff }}$ of low-mass mainsequences stars and the average mode lifetime $\langle\tau\rangle$ of the five most prominent modes:

$\langle\tau\rangle \propto T_{\text {eff }}^{-4}$

(Chaplin et al. 2009). This relation can obviously be of importance in the simulation of asteroseismic oscillations and the selection of targets. However, it should in any case not be a surprise that further development of the analysis techniques is required for stars whose properties differ substantially from those of the Sun. These developments will of course be inspired by the extensive data obtained from CoRoT and expected from Kepler. However, analysis of realistically simulated data for a variety of stellar parameters is also very valuable, ideally carried out in blind, so-called 'Hare and

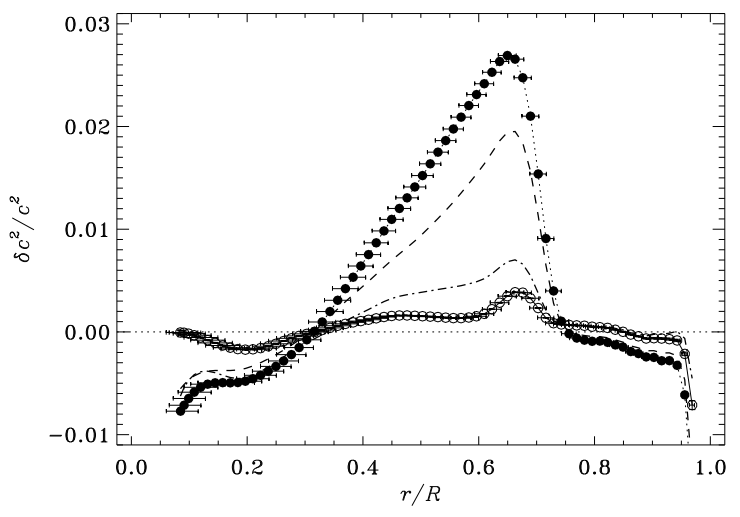

Fig. 12 Relative differences in squared sound speed between the Sun and three solar models, in the sense (Sun)(model), inferred from inversion of the so-called 'Best set' of observed frequencies (see Basu et al. 1997). The barely visible vertical bars mark $1 \sigma$ errors in the inferences, while the horizontal bars indicate the resolution of the inversion. Open symbols: Model S of Christensen-Dalsgaard et al. (1996); filled circles: corresponding model, but assuming the Asplund (2005) composition; dashed curve: corresponding model, but assuming the Asplund et al. (2009) composition; dot-dashed curve: model corresponding to Model S, but switching off electron screening (in the core this can hardly be distinguished from the dashed curve)

Hounds', investigations. Tools have been developed for such simulations (e.g., De Ridder et al. 2006), and they are being used in extensive analyses in the asteroFLAG project, with specific application to the data expected from the Kepler mission (Chaplin et al. 2008).

\section{Effects of model physics}

An ultimate goal of helio- and asteroseismology is obviously to relate the inferences to the physics of stellar interiors and hence to get a better understanding of the processes taking place in stars. Here we consider a few relevant examples, including some that were highlighted at the workshop, and consider effects on stellar structure that are, or may be, amenable to helio- and asteroseismic investigations.

\subsection{The solar sound speed}

The observed solar oscillation frequencies cover a broad range in spherical-harmonic degree. This has allowed inverse analyses to determine solar internal properties, in particular the sound speed, with high accuracy in most of the solar interior. Solar models computed a decade ago generally provided relatively good agreement with these inferences, with maximum relative deviations in the square of the sound speed well below 


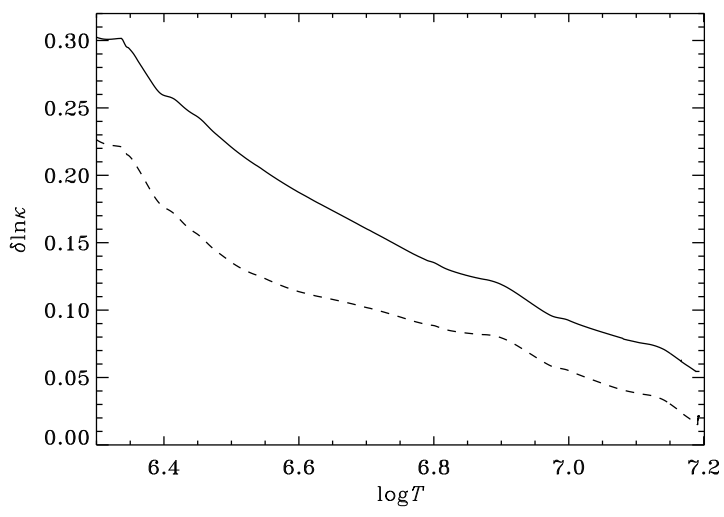

Fig. 13 Changes to the natural logarithm $\ln \kappa$ of the opacity, required to bring models with revised composition in accordance with Model S of Christensen-Dalsgaard et al. (1996). Solid curve: correction for the Asplund (2005) composition; dashed curve: correction for the Asplund et al. (2009) composition. (See Christensen-Dalsgaard et al. 2009)

1 per cent. A typical example is illustrated by the open symbols in Fig. 12, based on the so-called Model $S$ of Christensen-Dalsgaard et al. (1996); in common with many solar models of the epoch it assumed the Grevesse \& Noels (1993) solar composition. However, starting with Allende Prieto et al. (2001) reanalyses of solar spectral lines have led to substantial revisions in the determination of the surface abundances of, in particular, carbon, nitrogen and oxygen, leading to a reduction in the ratio $Z_{\mathrm{s}} / X_{\mathrm{s}}$ between the abundances by mass $Z_{\mathrm{s}}$ of heavy elements and $X_{\mathrm{s}}$ of hydrogen from 0.0245 to 0.0165 . This revision resulted from the use of hydrodynamical models of the solar atmosphere and the inclusion of NLTE effects in the analysis (for reviews, see Asplund 2005; Asplund et al. 2005). The main effect on solar modelling is a corresponding reduction in the opacity, leading to a substantial change in the sound speed in the radiative interior. The effect on the sound speed is illustrated by the filled symbols in Fig. 12 ${ }^{2}$ It is evident that this has led to a serious deterioration in the agreement between the solar models and the helioseismic inference.

This conflict between helioseismology and the solar models resulting from the revised composition was discussed in detail by Basu \& Antia (2008); possible solutions were reviewed by Guzik (2006), with the conclusion that none of the proposals were fully satisfactory.

${ }^{2}$ Here, and in the following results on solar modelling, the models correspond to Model S except in the specific property under investigation; the models have all been calibrated to solar radius, luminosity and the assumed value of $Z_{\mathrm{s}} / X_{\mathrm{s}}$ at solar age, by adjusting the mixing length and the initial composition.
Since the dominant effect on the models of the change in the composition arises from the resulting change in opacity, a simple solution would be to make corresponding changes to the intrinsic opacity (e.g., Bahcall et al. 2005). Christensen-Dalsgaard et al. (2009) estimated the opacity change required to restore the model computed with the old composition; the result is illustrated in Fig. 13. It is perhaps questionable if such a major change, of up to 30 per cent, is physically justifiable, although there are undoubtedly significant uncertainties in the opacity calculations.

Recently, Asplund et al. (2009) reconsidered the abundance determinations, using improved solar atmosphere models and a careful selection of atomic data and spectral lines (see also Grevesse et al. 2010, this volume). This resulted in a modest increase in the inferred abundances, such that $Z_{\mathrm{s}} / X_{\mathrm{s}}=0.0181$. The effect on the comparison between the resulting solar model and helioseismic inferences is illustrated by the dashed line in Fig. 12. Obviously the model is somewhat closer to the solar sound speed although the differences are still much larger than for the original model. The opacity change required to restore the original model is illustrated in Fig. 13, the maximal change, around 23 per cent, may still be larger than can reasonably be explained by errors in the opacity determinations.

Other uncertainties in stellar modelling may have a noticeable effect on the comparison with helioseismic inferences. An interesting example is the effect of electron screening on the nuclear reactions. This is normally treated in the Debye-Hückel approximation, according to a formulation developed by Salpeter (1954); here the formation of clouds of negative charge around the reacting nuclei leads to a reduction in the Coulomb repulsion and hence an increase in the reaction rates. However, it has been pointed out that the underlying mean-field approximation may be invalid under stellar conditions (see, for example, Shaviv 2004, for a review). As discussed by Mussack \& Däppen (2010, this volume) (see also Mao et al. 2009) this has motivated calculations of the properties of stellar plasmas using the techniques of molecular dynamics. The results indicate that the effects of electron screening are weaker than in the Salpeter formulation and that the interactions between the charged particles may in fact lead to a reduction in the reaction rates. To investigate the consequences for solar modelling we have computed a model where electron screening was simply switched off. The resulting sound-speed difference is illustrated by the dot-dashed line in Fig. 12, Although less dramatic than the effect of the change in composition, the change in the nuclear reactions, and the resulting change in the composition required to calibrate the model, leads 
to a definite deterioration in the agreement with the helioseismically inferred sound speed; such an effect of suppressing electron screening was already pointed out by Weiss et al. (2001).

\subsection{The onset of convective cores}

A major uncertainty in the modelling of intermediateand high-mass stars is the treatment of convective cores. Convective cores are typically found in stars of masses just a little higher than the mass of the Sun, thus including also stars where solar-like oscillations are expected. As discussed in Section 2.2 the detailed properties of the core are reflected in the small frequency separations and hence there are excellent prospects to use asteroseismology to constrain the modelling of convective cores. A detailed analysis of this was carried out by Popielski \& Dziembowski (2005), emphasizing the effect of the composition discontinuity caused by the growing convective core in stars of mass up to around $1.9 \mathrm{M}_{\odot}$.

Here we consider the effects of modifications to the microphysics and composition of the stellar interiors. The convective instability is caused by the increasing temperature sensitivity of nuclear reactions as the $\mathrm{CNO}$ cycle, with increasing stellar mass, becomes dominant in hydrogen burning. An additional contribution is the gradual conversion of ${ }^{16} \mathrm{O}$ to ${ }^{14} \mathrm{~N}$ through a branch of the $\mathrm{CNO}$ cycle; this increases the abundance of ${ }^{14} \mathrm{~N}$ which controls the overall rate of the energy generation from the CNO cycle and hence its importance, leading to the growth of the mass of the convective core.

It is obvious that the $\mathrm{CNO}$ cycle is sensitive to the abundances of carbon, nitrogen and oxygen. Abundances of solar-like stars are typically determined relative to the solar abundance, and hence the recent revisions of the solar abundances have a similar effect on the assumed abundances of stars and hence potentially on the onset of convective cores. In fact, VandenBerg et al. (2007) showed that the Asplund et al. (2005) abundances led to isochrones for the open cluster M67 which did not show a 'hook', resulting from the presence of a convective core. This was in contrast to models with the old composition, which showed a clear hook, and in apparent contradiction to observed colour-magnitude diagrams for the cluster. To investigate this further, we have considered the properties of convective cores as a function of stellar mass, characterizing them by the maximum mass reached by the core during central hydrogen burning. The models neglected diffusion and settling and used the NACRE (Angulo et al. 1999) nuclear parameters, but otherwise largely corresponded to Model S of Christensen-Dalsgaard et al. (1996). The

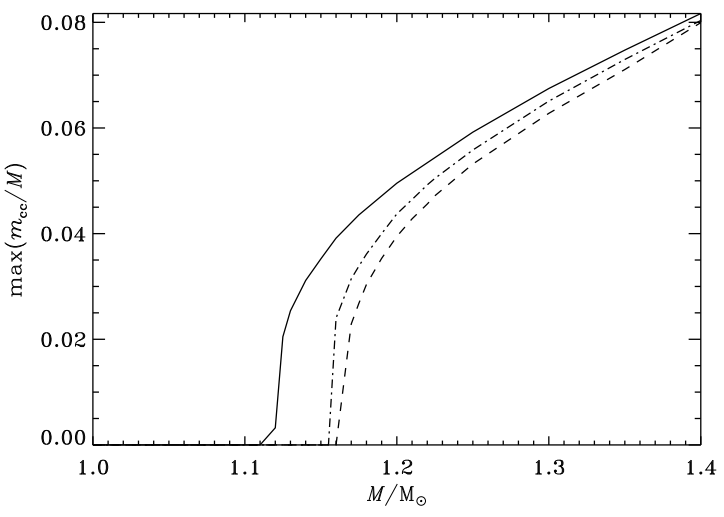

Fig. 14 Maximum fractional mass of the convective core during main-sequence evolution, as a function stellar mass in units of the solar mass. The models used the Angulo et al. (1999) nuclear parameters and included electron screening; diffusion and settling was not included. Solid line: reference model, with the Grevesse \& Noels (1993) composition, and initial hydrogen and heavy-element abundances given by $\left(X_{0}, Z_{0}\right)=(0.708,0.0196)$; dashed line: Asplund (2005) composition, and $\left(X_{0}, Z_{0}\right)=(0.730,0.0137)$, dot-dashed line: Asplund et al. (2009) composition, and $\left(X_{0}, Z_{0}\right)=$ $(0.722,0.0149)$

results are illustrated in Fig. 14. In agreement with VandenBerg et al. (2007) the mass at onset of the convective core increases by around $0.04 \mathrm{M}_{\odot}$ as a result of the Asplund et al. (2005) revision of the composition. The modest revision by Asplund et al. (2009) has only a slight effect, in comparison.

The presence of convective cores is obviously also sensitive to nuclear properties, particularly for the reaction ${ }^{14} \mathrm{~N}\left({ }^{1} \mathrm{H}, \gamma\right){ }^{15} \mathrm{O}$ which controls the rate of the dominant part of the CNO cycles. From new laboratory experiments and analyses, Angulo et al. (2005) found a large reduction in this rate. As shown in Fig. 15 this has a substantial effect on the minimum mass of stars with convective cores, increasing it by $0.06 \mathrm{M}_{\odot}$. On the other hand, since the CNO cycle makes a modest contribution to solar energy generation the effect on solar models is largely insignificant.

We have also considered the effect on the convective cores of switching off electron screening; the effect of electron screening increases with the charges of the interacting nuclei and hence turning it off decreases the relative importance of the $\mathrm{CNO}$ cycle, again delaying the onset of convective cores. This is also illustrated in Fig. 15, the effect is relatively modest.

Further investigations will be required to test the effects on the oscillation frequencies of these changes in the model properties, compared with other uncertainties in the treatment of convective cores, and the prospects for applying asteroseismic diagnostics, such 


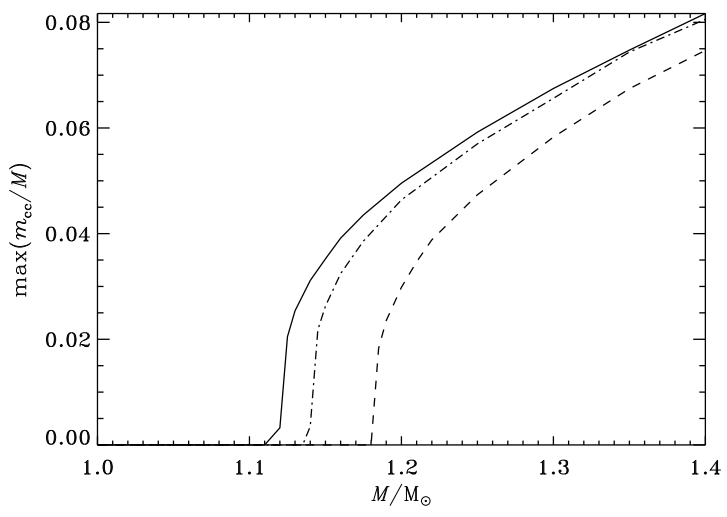

Fig. 15 As Fig. 14, all models having the Grevesse \& Noels (1993) composition. Solid line: reference model; dashed line: reduced ${ }^{14} \mathrm{~N}$ reaction rate (Angulo et al. 2005); dot-dashed line: no electron screening

as those proposed by Cunha \& Metcalfe (2007), to investigate them.

\section{Prospects for the future}

Asteroseismology is being revolutionized in the present period. As documented in a recently published special Volume 506 of Astronomy and Astrophysics, the initial results from the CoRoT mission are very promising, and at the time of writing the very early data from the Kepler mission are being analysed, through an intense and coordinated effort in the Kepler Asteroseismic Science Consortium, with a view towards publication in early 2010. Given the continuing operations of CoRoT and the very extensive data to be obtained with Kepler it is clear that coming years will see a huge effort in the analysis of asteroseismic data, with a corresponding increase in our information on and, one may hope, understanding of stellar properties. On a somewhat longer timescale there is hope that the PLATO mission Catala 2008) will provide detailed asteroseismic data on tens of thousands of stars.

Despite the impressive success of space-based asteroseismic investigations, there is still a need for groundbased observations. As found early in observations of the Sun (Harvey 1988) the intrinsic stellar noise is much higher, relative to the oscillations, in photometric observations than in observations of Doppler velocity. Velocity observations can be carried out from the ground, the main limiting factor being the access to the required instrumentation, at several sites to ensure continuous data and over a sufficient length of time. This motivates the establishment of dedicated facilities; an example is the Stellar Observations Network Group (SONG) project (Grundahl et al. 2009), aiming to establish a network of 8 observatories, with a suitable world-wide distribution, equipped with $1 \mathrm{~m}$ telescopes and high-resolution spectrographs to carry out asteroseismic Doppler-velocity observations. The proposal to set up the SIAMOIS facility for Doppler-velocity observations on Dome C in Antarctica (Mosser et al. 2008) is also very promising.

In parallel with these great observational strides there is a strong need for further development of the techniques for stellar modelling and the interpretation of the asteroseismic data. It is already clear that, not surprisingly, the oscillation properties of other stars show striking departures from the familiar properties of solar oscillations and that new techniques will be needed to optimize the analysis of the data and the use of the results for asteroseismic inferences. From the results of these analyses we can surely expect further surprises and identification of deficiencies in our stellar modelling, leading to new insights into the physics of stellar interiors.

Acknowledgements We are very grateful to Maria Pia Di Mauro for the organization of a very interesting workshop, and for a long and warm friendship. This work was supported by the Danish Natural Science Research Council and by the European Helio- and Asteroseismology Network (HELAS), a major international collaboration funded by the European Commission's Sixth Framework Programme. GH acknowledges support by the Austrian Science Fund (FWF project P21205). 


\section{References}

Aerts, C., Christensen-Dalsgaard, J., Kurtz, D. W.: Asteroseismology, Springer, Heidelberg (2009, in the press)

Allende Prieto, C., Lambert, D. L., Asplund, M.: Astrophys. J. 556, L63 (2001)

Angulo, C., Arnould, M., Rayet, M., et al.: Nucl. Phys. A 656, 3 (1999)

Angulo, C., Champagne, A. E., Trautvetter, H.-P.: Nucl. Phys. A 758, 391c (2005)

Appourchaux, T., Michel, E., Auvergne, M., et al.: Astron. Astrophys. 488, 705 (2008)

Arentoft, T., Kjeldsen, H., Bedding, T. R., et al.: Astrophys. J. 687, 1180 (2008)

Asplund, M.: Annu. Rev. Astron. Astrophys. 43, 481 (2005)

Asplund, M., Grevesse, N., Sauval, A. J.: In Cosmic Abundances as Records of Stellar Evolution and Nucleosynthesis, eds T. G. Barnes III, F. N. Bash, ASP Conf. Ser. 336, p. 25 (2005)

Asplund, M., Grevesse, N., Sauval, A. J., Scott, P.: Annu. Rev. Astron. Astrophys. 47, 481 (2009)

Baglin, A., Auvergne, M., Barge, P., Deleuil, M., Michel, E. and the CoRoT Exoplanet Science Team: In Proc. IAU Symp. 253, Transiting Planets, eds F. Pont, D. Sasselov, M. Holman, IAU and Cambridge Univ. Press, p. 71 (2009)

Bahcall, J. N., Basu, S., Pinsonneault, M., Serenelli, A. M.: Astrophys. J. 618, 1049 (2005)

Baker N., Gough D. O.: Astrophys. J. 234, 232 (1979)

Balmforth N. J.: Mon. Not. R. Astron. Soc. 255, 603 (1992a)

Balmforth N. J.: Mon. Not. R. Astron. Soc. 255, 632 (1992b)

Basu, S., Antia, H. M.: Astrophys. J. 606, L85 (2004)

Basu, S., Antia, H. M.: Phys. Rep. 457, 217 (2008)

Basu S., Antia H. M., Narasimha D.: Mon. Not. R. Astron. Soc. 267, 209 (1994)

Basu, S., Chaplin, W. J., Christensen-Dalsgaard, J., Elsworth, Y., Isaak, G. R., New, R., Schou, J., Thompson, M. J., Tomczyk, S.: Mon. Not. R. Astron. Soc. 292, 243 (1997)

Basu, S., Christensen-Dalsgaard, J., Thompson, M. J.: In Proc. 1st Eddington Workshop, 'Stellar Structure and Habitable Planet Finding', eds F. Favata, I. W. Roxburgh and D. Galadí-Enríquez, ESA SP-485, ESA Publications Division, Noordwijk, The Netherlands, p. 249 (2002)

Basu S., Chaplin W. J., Elsworth Y., New A. M., Serenelli G., Verner G. A.: Astrophys. J., 655, 660 (2007)

Bazot, M., Bouchy, F., Kjeldsen, H., Charpinet, S., Laymand, M., Vauclair, S.: Astron. Astrophys. 470, 295 (2007)

Bedding, T. R., Kjeldsen, H.: In Proc. $14^{\text {th }}$ Cambridge Workshop on Cool Stars, Stellar Systems, and the Sun, ed. G. T. van Belle, ASP Conf. Ser. 384, p. 21 (2008)

Bedding, T. R., Kjeldsen, H., Arentoft, T., et al.: Astrophys. J. 663, 1315 (2007)

Bedding, T. R., Kjeldsen, H., Bouchy, F., Bruntt, H., Butler, R. P., Buzasi, D. L., Christensen-Dalsgaard, J., Frandsen, S., Lebrun, J.-C., Martić, M., Schou. J.: Astron. Astrophys. 432, L43 (2005)

Bedding, T. R., Kjeldsen, H., Butler, R. P., McCarthy, C., Marcy, G. W., O'Toole, S. J., Tinney, C. G., Wright, J. T.: Astrophys. J. 614, 380 (2004)
Benomar, O., Appourchaux, T., Baudin, F.: Astron. Astrophys. 506, 15 (2009)

Böhm-Vitense, E.: Z. Astrophys. 46, 108 (1958)

Borucki, W., Koch, D., Bathalha, N., et al.: In Proc. IAU Symp. 253, Transiting Planets, eds F. Pont, D. Sasselov, M. Holman, IAU and Cambridge Univ. Press, p. 289 (2009)

Bouchy, F., Carrier, F.: Astron. Astrophys. 374, L5 (2001)

Bouchy, F., Carrier, F.: Astron. Astrophys. 390, 205 (2002)

Brown, T. M., Gilliland, R. L., Noyes, R. W., Ramsey, L. W.: Astrophys. J. 368, 599 (1991)

Bruntt, H.: Comm. in Asteroseismology 150, 326 (2007)

Butler, R. P., Bedding, T. R., Kjeldsen, H., McCarthy, C., O'Toole, S. J., Tinney, C. G., Marcy, G. W., Wright, J. T.: Astrophys. J. 600, L75 (2004)

Buzasi, D. L., Bruntt, H., Bedding, T. R., et al.: Astrophys. J. 619, 1072 (2005)

Carrier, F., Bourban, G.: Astron. Astrophys. 406, L23 (2003)

Carrier, F., Eggenberger, P., Bouchy, F.: Astron. Astrophys. 434, 1085 (2005)

Catala, C., and the PLATO consortium: In Proc. HELAS II International Conference: Helioseismology, Asteroseismology and the MHD Connections, eds L. Gizon, M. Roth, J. Phys.: Conf. Ser. 118, 012040 (2008)

Chaplin, W. J., Appourchaux, T., Arentoft, T., et al.: Astron. Nach. 329, 549 (2008)

Chaplin, W. J., Houdek, G., Karoff, C., Elsworth, Y., New, R.: Astron. Astrophys. 500, L21 (2009)

Christensen-Dalsgaard, J.: Mon. Not. R. Astron. Soc. 190, 765 (1980)

Christensen-Dalsgaard J.: In Proc. Workshop Space research prospects in stellar activity and variability, eds A. Mangeney, F. Praderie, Obs. Paris-Meudon, p. 11 (1984)

Christensen-Dalsgaard J.: In Proc. GONG 1992: Seismic investigation of the Sun and stars, ed. T. M. Brown, ASP Conf. Ser. 42, p. 347 (1993)

Christensen-Dalsgaard, J.: Rev. Mod. Phys. 74, 1073 (2002)

Christensen-Dalsgaard, J.: Sol. Phys. 220, 137 (2004)

Christensen-Dalsgaard J., Frandsen S.: Sol. Phys. 82, 469 (1983)

Christensen-Dalsgaard, J., Gough, D. O.: Nature 288, 544 (1980)

Christensen-Dalsgaard, J., Pérez Hernández, F.: Mon. Not. R. Astron. Soc. 257, 62 (1992)

Christensen-Dalsgaard, J., Thompson, M. J.: Mon. Not. R. Astron. Soc. 284, 527 (1997)

Christensen-Dalsgaard J., Bedding T., Houdek G., Kjeldsen H., Rosenthal C. S., Trampedach R., Monteiro M. J. P. F. G., Nordlund, Å.: In Proc. IAU Colloq. 155, Astrophysical Applications of Stellar Pulsation, eds R. S. Stobie, P. A. Whitelock, PASP 83, p. 447 (1995)

Christensen-Dalsgaard, J., Däppen, W., Ajukov, S. V., et al.: Science 1286 (1996)

Christensen-Dalsgaard, J., Arentoft, T., Brown, T. M., Gilliland, R. L., Kjeldsen, H., Borucki, W. J., Koch, D.: In Proc. HELAS II International Conference: Helioseismology, Asteroseismology and the MHD Connections, eds L. Gizon, M. Roth, J. Phys.: Conf. Ser. 118, 012039 (2008) 
Christensen-Dalsgaard, J., Di Mauro, M. P., Houdek, G., Pijpers, F.: Astron. Astrophys. 494, 205 (2009)

Cunha, M. S., Metcalfe, T. S.: Astrophys. J. 666, 413 (2007)

De Ridder, J., Arentoft, T., Kjeldsen, H.: Mon. Not. R. Astron. Soc. 365, 595 (2006)

De Ridder, J., Barban, C., Baudin, F., et al.: Nature 459, 398 (2009)

Di Mauro, M. P., Christensen-Dalsgaard, J., Kjeldsen, H., Bedding, T. R., Paternò, L.: Astron. Astrophys. 404, 341 (2003)

Dupret, M.-A., Belkacem, K., Samadi, R., et al.: Astron. Astrophys. 506, 57 (2009)

Eggenberger, P., Charbonnel, C., Talon, S., Meynet, G., Maeder, A., Carrier, F., Bourban, G.: Astron. Astrophys. 417, 235 (2004)

Fletcher, S. T., Chaplin, W. J., Elsworth, Y., Schou, J., Buzasi, D.: Mon. Not. R. Astron. Soc. 371, 935 (2006)

Gaulme, P., Appourchaux, T., Boumier, P.: Astron. Astrophys. 506, 7 (2009)

Goldreich P., Keeley D. A.: Astrophys. J. 212, 243 (1977)

Gough D. O.: Astrophys. J. 214, 196 (1977a)

Gough D. O.: In Proc. IAU Colloq. No. 38, Problems of Stellar Convection, eds E. A. Spiegel, J.-P. Zahn, Lecture Notes in Physics 71, Springer-Verlag, Berlin, p. 15 (1977b)

Gough, D. O.: In Nonradial and Nonlinear Stellar Pulsation, eds H. A. Hill, W. A. Dziembowski, Lecture Notes in Physics 125, Springer-Verlag, Berlin, p. 273 (1980)

Gough, D. O.: In Proc. Hydrodynamic and magnetohydrodynamic problems in the Sun and stars, ed. Y. Osaki, University of Tokyo, Tokyo, p. 117 (1986)

Gough, D. O.: In Progress of Seismology of the Sun and Stars, Lecture Notes in Physics 367, eds Y. Osaki, H. Shibahashi, Springer Verlag, p. 283 (1990)

Gough, D. O.: In Astrophysical fluid dynamics, Les Houches Session XLVII, eds J.-P. Zahn, J. Zinn-Justin, Elsevier, Amsterdam, p. 399 (1993)

Gough D. O.: In Astrophysical Ages and Timescales, eds T. von Hippel, C. Simpson, N. Manset, ASP Conf. Ser. 245, p. 31 (2001)

Gough D. O.: In Stellar structure and habitable planet finding, eds F. Favata, I. W. Roxburgh, D. Galadi, ESA SP485, ESA Publications Division, Noordwijk, p. 65 (2002)

Gough, D. O., Kosovichev, A. G.: In Proc. IAU Colloq. 137: Inside the stars, eds A. Baglin, W. W. Weiss, ASP Conf. Ser. 40, p. 541 (1993)

Gough, D. O., Kosovichev, A. G., Toomre, J., et al.: Science 272, 1296 (1996)

Grec, G., Fossat, E., Pomerantz, M.: Sol. Phys. 82, 55 (1983)

Grevesse, N., Noels, A.: In Origin and evolution of the Elements, eds N. Prantzos, E. Vangioni-Flam, M. Cassé Cambridge Univ. Press, Cambridge, p. 15 (1993)

Grevesse, N., Asplund, M., Sauval, J., Scott, P., Klein, O.: Astrophys. Space Sci. (2010, this volume)

Grundahl, F., Christensen-Dalsgaard, J., Kjeldsen, H., Jørgensen, U. G., Arentoft, T., Frandsen, S., Kjærgaard, P.: In Proc. GONG2008/SOHO21 meeting: Solar-stellar Dynamos as revealed by Helio- and Asteroseismology, eds M. Dikpati, T. Arentoft, I. González Hernández, C. Lindsey, F. Hill, ASP Conf. Ser., [arXiv:0908.0436v1 [astro-ph.SR]] (2009, in the press)
Guzik, J. A.: In Proc. SOHO 18 / GONG 2006 / HELAS I Conf. Beyond the spherical Sun, ed. K. Fletcher, ESA SP624, ESA Publications Division, Noordwijk, The Netherlands (2006)

Guzik, J. A.: Mem. Soc. Astron. Italiana 79, 481 (2008)

Harvey, J. W.: In Proc. IAU Symposium No 123, Advances in helio- and asteroseismology, eds J. ChristensenDalsgaard, S. Frandsen, Reidel, Dordrecht, p. 497 (1988)

Houdek G.: Ph.D. Thesis, Pulsation of Solar-Type stars, University of Vienna, Vienna (1996)

Houdek, G.: In Proc. SOHO 18 / GONG 2006 / HELAS I Conf. Beyond the spherical Sun, ed. K. Fletcher, ESA SP624, ESA Publications Division, Noordwijk, The Netherlands (2006)

Houdek G., Gough D. O.: Mon. Not. R. Astron. Soc. 375, 861 (2007)

Houdek G., Gough D. O.: In Proc. IAU Symp. 252, The Art of Modelling Stars in the 21st Century, eds L. Deng, K. L. Chan, C. Chiosi, Cambridge Univ. Press, Cambridge, p. 149 (2008)

Houdek, G., Balmforth, N. J., Christensen-Dalsgaard, J., Gough, D. O.: Astron. Astrophys. 351, 582 (1999)

Kjeldsen, H., Bedding, T. R., Viskum, M., Frandsen, S.: Astron. J. 109, 1313 (1995)

Kjeldsen, H., Bedding, T. R., Baldry, I. K., et al.: Astron. J. 126, 1483 (2003)

Kjeldsen, H., Bedding, T. R., Butler, R. P., ChristensenDalsgaard, J., Kiss, L. L., McCarthy, C., Marcy, G. W., Tinney, C. G., Wright, J. T.: Astrophys. J. 635, 1281 (2005)

Kjeldsen, H., Bedding, T. R., Christensen-Dalsgaard, J.: Astrophys. J. 683, L175 (2008)

Kjeldsen, H., Bedding, T. R., Christensen-Dalsgaard, J.: In Proc. IAU Symp. 253, Transiting Planets, eds F. Pont, D. Sasselov, M. Holman, IAU and Cambridge Univ. Press, 309 (2009)

Lebreton, Y., Monteiro, M. J. P. F. G., Montalbán, J., et al.: Astrophys. Space Sci. 316, 1 (2008a)

Lebreton, Y., Montalbán, J., Christensen-Dalsgaard, J., Roxburgh, I. W., Weiss, A.: Astrophys. Space Sci. 316, 187 (2008b)

Li, L. H., Robinson, F. J., Demarque, P., Sofia, S.: Astrophys. J. 567, 1192 (2002)

Mao, D., Mussack, K., Däppen, W.: Astrophys. J. 701, 1204 (2009)

Matthews, J. M.: Comm. in Asteroseismology 150, 333 (2007)

Matthews, J. M., Kuschnig, R., Guenther, D. B., Walker, G. A. H., Moffat, A. F. J., Rucinski, S. M., Sasselov, D., Weiss, W. W.: Nature 430, 51 (Erratum: Nature 430, 921) (2004)

Michel, E., Baglin, A., Weiss, W. W., et al.: Comm. in Asteroseismology 156, 73 (2008a)

Michel, E., Baglin, A., Auvergne, M., et al.: Science 322, 558 (2008b)

Miglio, A., Montalbán, J.: Astron. Astrophys. 441, 615 (2005)

Monteiro M. J. P. F. G., Thompson M. J.: In Proc. IAU Symp. 185, New Eyes to see inside the Sun and Stars, eds F.-L. Deubner, J. Christensen-Dalsgaard, D. W. Kurtz, Kluwer, Dordrecht, p. 317 (1998) 
Monteiro M. J. P. F. G., Thompson M. J.: Mon. Not. R. Astron. Soc. 361, 1187 (2005)

Mosser, B., Appourchaux, T., Catala, C., Buey, J.-T. and the SIAMOIS team: In Proc. HELAS II International Conference: Helioseismology, Asteroseismology and the MHD Connections, eds L. Gizon, M. Roth, J. Phys.: Conf. Ser. 118, 012042 (2008)

Moya, A., Christensen-Dalsgaard, J., Charpinet, S., et al.: Astrophys. Space Sci. 316, 231 (2008)

Mussack, K., Däppen, W.: Astrophys. Space Sci. (2010, this volume)

Osaki, Y.: Publ. Astron. Soc. Jpn. 27, 237 (1975)

Otí Floranes, H., Christensen-Dalsgaard, J., Thompson, M. J.: Mon. Not. R. Astron. Soc. 356, 671 (2005)

Popielski, B. L., Dziembowski, W. A.: Acta Astron. 55, 177 (2005)

Rosenthal, C. S., Christensen-Dalsgaard, J., Houdek, G., Monteiro, M. J. P. F. G., Nordlund, Å., Trampedach, R.: In Proc. 4th SOHO Workshop: Helioseismology, eds J. T. Hoeksema, V. Domingo, B. Fleck, B. Battrick, ESA SP-376, vol.2, ESTEC, Noordwijk, p. 459 (1995)

Rosenthal, C. S., Christensen-Dalsgaard, J., Nordlund, Å., Stein, R. F., Trampedach, R.: Astron. Astrophys. 351, 689 (1999)

Roxburgh, I. W.: In Proc. 2nd Eddington workshop, "Stellar structure and habitable planet finding", ESA SP-538, eds F. Favata, S. Aigrain, ESA Publications Division, Noordwijk, The Netherlands, p. 23 (2004)

Roxburgh, I. W.: Astron. Astrophys. 434, 665 (2005)

Roxburgh, I. W.: Astron. Astrophys. 493, 185 (2009)

Roxburgh, I. W., Vorontsov, S. V.: Mon. Not. R. Astron. Soc. 267, 297 (1994)

Roxburgh, I. W., Vorontsov, S. V.: Mon. Not. R. Astron. Soc. 317, 141 (2000)

Roxburgh, I. W., Vorontsov, S. V.: Astron. Astrophys. 411, 215 (2003)

Roxburgh, I. W., Vorontsov, S. V.: Mon. Not. R. Astron. Soc. 379, 801 (2007)

Salpeter, E. E.: Austr. J. Phys. 7, 373 (1954)

Shaviv, G.: In Equation-of-State and Phase-Transition Issues in Models of Ordinary Astrophysical Matter, eds V. Čelebonović, W. Däppen, D. Gough, AIP Conf. Proc. 731, AIP, Melville, New York, p. 67 (2004)

Stello, D., Chaplin, W. J., Bruntt, H., et al.: Astrophys. J. 700, 1589 (2009)

Straka, C. W., Demarque, P., Guenther, D. B., Li, L., Robinson, F. J.: Astrophys. J. 636, 1078 (2006)

Tassoul M.: Astrophys. J. Suppl. Ser. 43, 46 (1980)

Ulrich, R. K.: Astrophys. J. 306, L37 (1986)

Vandakurov, Y. V.: Astron. Zh. 44, 786 (English translation: Soviet Astron. 11, 630) (1967)

VandenBerg, D. A., Gustafsson, B., Edvardsson, B., Eriksson, K., Ferguson, J.: Astrophys. J. 666, L105 (2007)

Walker, G., Matthews, J., Kuschnig, R., et al.: Publ. Astron. Soc. Pac. 115, 1023 (2003)

Weiss, A., Flaskamp, M., Tsytovich, V. N.: Astron. Astrophys. 371, 1123 (2001)

This manuscript was prepared with the AAS LATEX macros v5.2. 